Studie sieht Roche bei Websites auf Platz eins

Das Pharma-Unternehmen Roche hält im Internet das beste Angebot für medizinische Fachkreise vor. Das hat die Studie Benchmarking Websites Pharma 2010 ergeben. Die Studie ist jetzt zum zehnten Mal vom Unternehmen Consert Online Service Pharma vorgelegt worden. Auf den Plätzen zwei bis fünf folgen mit ihren WebAngeboten für Fachkreise die Unternehmen Bayer Vital, Hexal, GlaxoSmithKline und MSD Sharp \& Dohme. Mehr als 4.000 medizinische Websites von Pharma-Unternehmen in insgesamt 18 Indikationsbereichen sind anhand eines einheitlichen Rasters in die Bewertung eingeflossen. ger

\section{DGN bietet neuen}

\section{KV-SafeNet-Router an}

Das Deutsche Gesundheitsnetz (DGN), das zum IT-Anbieter DGN Service GmbH gehört, hat einen neuen Zugangsrouter für das KV-SafeNet entwickelt. Das Eigenprodukt "GUSbox ${ }^{\circledast} \mathrm{S}^{\prime \prime}$ löse die bislang vom DGN konfigurierte FRITZ!Box als KV-SafeNet-Router ab, meldet das Unternehmen, und werde als Mietgerät für monatlich 4,90 Euro angeboten. Im Mietpreis enthalten seien die Firmware, Sicherheits-Updates sowie ein 24-Stunden-Austausch-Service im Fall einer defekten Box. Zudem soll die GUSbox ${ }^{\circledR}$ S im zweiten Quartal mit zusätzlichen Diensten, etwa einem eingebauten D2D-Dienst, ausgestattet werden.

promedico relauncht eigene Homepage

Die promedico Computer für Medizin $\mathrm{GmbH}$ hat einen neuen Internetauftritt (www.promedico.com). Die Inhalte der Webseite reichten von aktuellen Informationen über Produktbeschreibungen bis hin zu Serviceangebote für die Anwender einer promedico-Software, so das Unternehmen. Beispielsweise sollen Besucher der Seite zu aktuellen Themen wie den Ambulanten Kodierrichtlinien umfassende Informationen erhalten. Außerdem sollen promedico-Kunden Hilfe, Unterstützung und Informationen zu Produktneuheiten finden. Interviews mit und Berichte von promedico-Anwendern sollen den Praxisteams Aufschluss darüber geben, welche Erfahrungen Kollegen mit promedico-Lösungen gesammelt haben. Zudem sollen regelmäßige und aktuelle Informationen auch über einen Newsletter bezogen werden können.

\title{
Health-Apps auf dem Weg in die Praxen
}

\author{
Mobile Programme für Handys entwickeln sich zu einem enormen \\ Geschäft. Die Anwendungen haben nicht nur Unterhaltungswert. \\ Sie können auch den ärztlichen Alltag erleichtern.
}

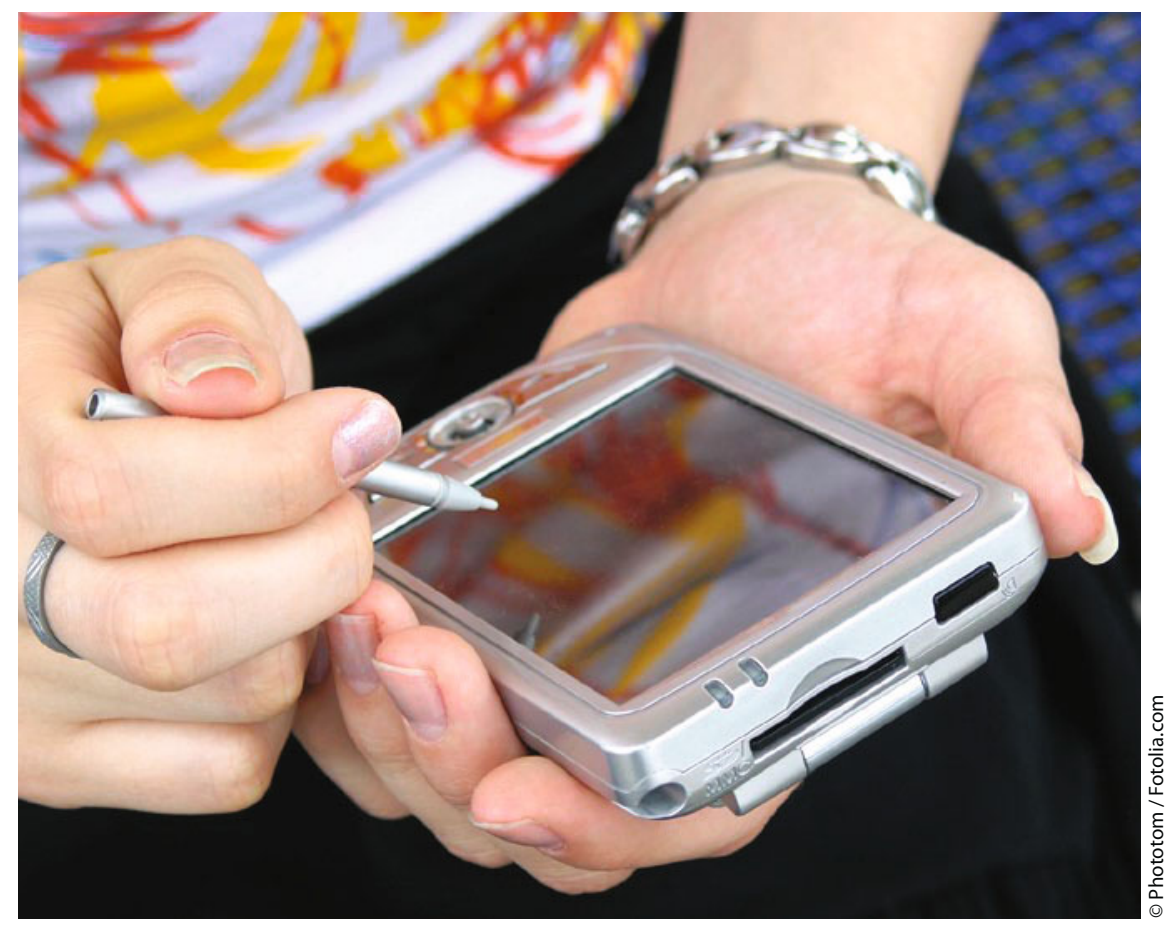

Mit Smartphones lässt sich mehr als der nächste Arzt suchen. Manche App hilft sogar bei der Diagnose.

$\mathrm{M}$ obile Applikationen - sogenannte Apps - gehören zu den Hauptattraktionen von Smartphones wie dem iPhone oder Handys mit Android-Betriebssystem. Die kleinen Software-Programme können Ärzte und Patienten aus dem Internet auf das Handy herunterladen und direkt nach der Installation anwenden. Und gerade der Gesundheitssektor soll künftig von den Programmen profitieren.

$67 \%$ der Unternehmen erwarten im medizinischen Bereich laut einer internationalen Studie des Marktforschungsunternehmens reseach2guidance, dass mobile Gesundheitsanwendungen in den kommenden fünf Jahren Ärzte und me- dizinisches Fachpersonal zunehmend unterstützen werden. Die GesundheitsApps sollen dabei nicht nur die Prävention und Fitness der Patienten fördern. Sie könnten auch die Versorgung chronisch Kranker verbessern, heißt es in der Studie, die jüngst vom Bundesverband Informationswirtschaft, Telekommunikation und neue Medien e. V. (BITKOM) vorgestellt wurde. Für die Studie wurden weltweit 231 Unternehmen aus dem Gesundheitsbereich befragt.

Eines der Haupteinsatzgebiete von „Health-Apps“ könnte dabei die Unterstützung von Diabetes-Patienten sein. Denn die direkten Kosten pro Diabetespatient in westlichen Ländern wie 